\title{
Use of Acellular Dermal Matrix is Comparable to Expander Based Breast Reconstructions for Post Operative Physiotherapy Requirements
}

\section{Kiernan $\mathrm{T}^{*}$ and Martin L}

University Hosptial Aintree, Lower Lane, Fazakerley, Liverpool, England, UK

\begin{abstract}
Currently all suitable women who require mastectomy for Breast Cancer should be offered breast reconstruction either as an immediate or delayed procedure. Various forms of breast reconstruction are available but since the introduction of Acellular Dermal Matrixes (ADMs), implant based reconstruction has become more popular. Implant based reconstruction has the advantages of being a relatively short operation with minimal morbidity compared to myocutaneous flap based reconstructions. ADMs are thought to improve the cosmetic outcomes of implant based reconstructions by allowing a more natural breast mound to be created. They also provide a one stage procedure as opposed to expander based reconstructions that require several inflations in clinic and a second operation to exchange the expander to a silicone implant. Most studies on the use of ADMs have been focused on cosmesis and financial or logistical benefits. To date there have been no studies on whether the use of ADM has an effect on post operative physiotherapy requirements. This study assessed the number of physiotherapy sessions required for women, who had undergone expander or ADM assisted breast reconstruction, to regain full shoulder mobility. There was no significant difference between the two procedures for physiotherapy requirements, with greater significance on the type of concomitant axillary surgery performed.
\end{abstract}

\section{Introduction}

In 2002, the National Institute for Health and Clinical Excellence (NICE) recommended that "reconstruction should be available [to all women with breast cancer] at the initial surgical operation [1]". Following this there has been an increase in the rate of immediate breast reconstructions either as a myocutaneous flap or with the use of an expander placed in a subpectoral pocket. The more recent introduction of Acellular Dermal Matrix (ADM) has allowed surgeons to provide a straight to implant reconstruction. The $\mathrm{ADM}$ is sutured to the inferior free edge of Pectoralis major, following disconnection from the chest wall, to form a large subpectoral pocket in which a silicone implant can be fitted. This provides a one stage procedure as opposed to expander based reconstructions that require a second operation to exchange the expander to an implant. Anecdotal advantages of the use of ADM has been improved cosmesis, reduction in post operative pain, precise control of the inframammary fold, faster time to completion of reconstruction, improved lower pole expansion, decreased rate of revision and reduced burden on Breast clinic resources. A recent review on the evidence behind these perceived advantages has shown paucity in data and a lack of convincing evidence [2].

Over the year 2012-2013 there was a perceived advantage of the use of ADM over expanders with regards to post operative pain and the need for physiotherapy treatment. To evaluate this we conducted a retrospective study of patients undergoing implant based breast reconstruction and the amount of physiotherapy treatments required post operatively to gain full shoulder function.

\section{Study Aim}

The aim of this study was to answer the question 'Does using ADM's reduce the amount of physiotherapy sessions required post operatively?'

\section{Methods}

Women undergoing expander or ADM breast reconstructions in 2012 were identified from physiotherapy notes. Patients who had myocutaneous flaps, dermal flaps or surgery involving both expander and ADM were excluded. Data collected included patient age, extent of axillary surgery, indication for mastectomy, implant or expander volumes, requirement for chemoradiotherapy, complications and number of physiotherapy treatments required to get to full range of shoulder movement.

\section{Patient Characteristics}

Over the 12 month period 29 patients who had undergone expander or ADM assisted breast reconstructions were identified. Six patients were excluded from analysis; four had undergone ADM with expander reconstruction, one had treatment in another hospital and one did not attend her physiotherapy appointment. The mean patient age was 51 years (range 38-65) with no significant difference between the two groups, 51 and 52 years for ADM and expander respectively. Out of the 23 patients included in analysis, 19 had the surgery for cancer treatment, two reconstructions were delayed and two were risk reducing. Ten patients underwent axillary node clearance at the time of the reconstruction, 9 had sentinel lymph node biopsy and four did not require any axillary surgery. There were no implant losses in this cohort of patients during the study period.

\section{Results}

Median values were used for comparison as the data was skewed by one patient in the expander group requiring 10 sessions and one patient in the ADM group requiring 7 sessions. The numbers within this study group were deemed too low for formal statistical analysis.

In the ADM group five (56\%) patients underwent axillary node clearance, three (33\%) had a sentinel lymph node biopsy and one (11\%) did not require any axillary treatment. This group required a median of two physiotherapy sessions.

*Corresponding author: Kiernan T, University Hosptial Aintree, Lower Lane, Fazakerley, Liverpool, England, L9 7AL, UK, E-mail: TAMARA.KIERNAN2@aintree.nhs.uk

Received April 26, 2013; Accepted July 01, 2013; Published July 09, 2013

Citation: Kiernan T, Martin L (2013) Use of Acellular Dermal Matrix is Comparable to Expander Based Breast Reconstructions for Post Operative Physiotherapy Requirements. Surgery Curr Res 3: 136. doi:10.4172/2161-1076.1000136

Copyright: @ 2013 Kiernan T, et al. This is an open-access article distributed under the terms of the Creative Commons Attribution License, which permits unrestricted use, distribution, and reproduction in any medium, provided the original author and source are credited. 
Citation: Kiernan T, Martin L (2013) Use of Acellular Dermal Matrix is Comparable to Expander Based Breast Reconstructions for Post Operative Physiotherapy Requirements. Surgery Curr Res 3: 136. doi:10.4172/2161-1076.1000136

Page 2 of 2

\begin{tabular}{|c|c|c|c|c|c|c|c|c|c|}
\hline Age & Indication & Axilla & Method & Initial vol & End vol & Chemo & Radio & Complications & No. sessions \\
\hline 62 & cancer & anc & $\mathrm{adm}$ & 440 & 440 & $y$ & $\mathrm{y}$ & $\mathrm{n}$ & 1 \\
\hline 46 & cancer & anc & adm & 390 & 390 & $y$ & $y$ & $\mathrm{n}$ & 2 \\
\hline 39 & cancer & anc & adm & 420 & 420 & $\mathrm{y}$ & $y$ & Neutropenia & 3 \\
\hline 43 & cancer & anc & adm & 375 & 375 & $y$ & $y$ & $\mathrm{n}$ & 7 \\
\hline 65 & cancer & sb+anc & adm & 310 & 310 & $y$ & $y$ & $\mathrm{n}$ & 0 \\
\hline 50 & cancer & slnb & adm & 310 & 310 & $\mathrm{n}$ & $\mathrm{n}$ & $\mathrm{n}$ & 0 \\
\hline 65 & cancer & $\operatorname{sinb}$ & $\mathrm{adm}$ & 420 & 420 & $\mathrm{n}$ & $\mathrm{n}$ & $\mathrm{n}$ & 2 \\
\hline 50 & cancer & $\operatorname{sinb}$ & adm & 335 & 335 & $\mathrm{n}$ & $\mathrm{n}$ & $\mathrm{n}$ & 2 \\
\hline 38 & $\mathrm{rr}$ & & adm & 450 & 450 & $\mathrm{n}$ & $\mathrm{n}$ & $\mathrm{n}$ & 1 \\
\hline 53 & cancer & anc & $\exp$ & 150 & 500 & $y$ & $y$ & $\mathrm{n}$ & 2 \\
\hline 49 & cancer & sb+anc & $\exp$ & 150 & 600 & $y$ & $y$ & seroma & 1 \\
\hline 61 & cancer & sb+anc & $\exp$ & 200 & 600 & $y$ & $\mathrm{n}$ & $\mathrm{n}$ & 1 \\
\hline 48 & cancer & sb+anc & $\exp$ & 200 & 400 & $y$ & $y$ & $\mathrm{n}$ & 3 \\
\hline 57 & cancer & sb+anc & $\exp$ & 300 & 650 & $y$ & $y$ & $\mathrm{n}$ & 10 \\
\hline 60 & cancer & $s \ln b$ & $\exp$ & 200 & 500 & $\mathrm{n}$ & $\mathrm{n}$ & $\mathrm{n}$ & 0 \\
\hline 52 & cancer & slnb & $\exp$ & 100 & 300 & $\mathrm{n}$ & $\mathrm{n}$ & $\mathrm{n}$ & 1 \\
\hline 47 & cancer & slnb & $\exp$ & 100 & 600 & $\mathrm{n}$ & $\mathrm{n}$ & $\mathrm{n}$ & 2 \\
\hline 54 & cancer & slnb & $\exp$ & 300 & 500 & $\mathrm{n}$ & $\mathrm{n}$ & $\mathrm{n}$ & 5 \\
\hline 41 & cancer & slnb & $\exp$ & 150 & 300 & $y$ & $\mathrm{n}$ & $\mathrm{n}$ & 1 \\
\hline 41 & cancer & $\operatorname{sinb}$ & $\exp$ & 150 & 400 & $y$ & $\mathrm{n}$ & $\mathrm{n}$ & 1 \\
\hline 49 & delayed & & $\exp$ & 300 & 600 & $\mathrm{n}$ & $\mathrm{n}$ & $\mathrm{n}$ & 1 \\
\hline 46 & $\mathrm{rr}$ & & $\exp$ & 150 & 500 & $\mathrm{n}$ & $\mathrm{n}$ & seroma & 1 \\
\hline 65 & delayed & & $\exp$ & 200 & 400 & $\mathrm{n}$ & $\mathrm{n}$ & $\mathrm{n}$ & 1 \\
\hline
\end{tabular}

Table 1: Anc = axillary node clearance, $\operatorname{SInb}=$ sentinel lymph node biopsy.

In the expander group five (36\%) patients underwent axillary node clearance, 6 (43\%) had a sentinel lymph node biopsy and three (21\%) did not require any axillary treatment. This group required a median of one physiotherapy session.

As the extent of axillary surgery has a significant effect on patients post operative comfort and shoulder mobilisation we calculated how many treatment sessions were required for the different types of axillary surgery. Patients who underwent axillary node clearance had a median of two physiotherapy sessions; patients who had a sentinel lymph node biopsy or no axillary surgery required only one session.

Patients who required adjuvant chemo and radiotherapy underwent a median of two physiotherapy sessions; patients that did not require any adjuvant treatment underwent one session. This may be a reflection of the effect of axillary surgery as all the patients in the adjuvant group had axillary node dissection and the patients in the no adjuvant group had sentinel lymph node biopsy only.

Breaking this down into the two reconstruction techniques gave a median of two physiotherapy sessions for patients with ADM reconstruction who had undergone adjuvant chemo and radiotherapy following axillary node clearance and 2.5 sessions for those with the expander. This small difference may be explained by the observation that three of the four patients in the expander group had undergone two axillary procedures - a sentinel lymph node biopsy followed by axillary node clearance. This is in comparison with only one of the five patients in the ADM group having two axillary procedures; the rest had immediate axillary node clearance. For patients who did not require any adjuvant therapy the ADM group had a median of 1.5 physiotherapy sessions and the expander group one. Table 1 depicts the collected data.

\section{Discussion}

The small sample size in this study was unable to demonstrate a clear difference in any advantage or disadvantage of the use of ADM or expander based reconstructions and the number of physiotherapy sessions required to gain full shoulder mobility post operatively. There was a suggestion that the extent of axillary surgery was the most important factor when assessing shoulder mobility but it is likely that this is multifactorial with adjuvant cancer treatments also having played a role.

At present there are no studies addressing the issue of shoulder mobility following ADM assisted breast reconstruction. In fact, as stated in a recent review, the evidence for the perceived advantages of ADM assisted reconstruction over expander reconstruction is currently rather sparse [2]. The evidence that is present is concentrated on the cosmetic outcomes rather than patient morbidity.

Until larger studies are performed it is not clear if ADM assisted reconstruction is superior to expander reconstruction with regards to physiotherapy requirements, however from this study it seems that these techniques are comparable.

\section{Acknowledgements}

J Mcateer, Senior Physiotherapist, University Hospital Aintree.

\section{References}

1. National Institute for Clinical Excellence (NICE) (2002) Guidance on cance services. Improving outcomes in breast cancer- manual update. London: NICE.

2. Nguyen T, Carey J, Wong A (2011) Use of human acellular dermal matrix in implant-based breast reconstruction: Evaluating the evidence. J Plast Reconstr Aesthet Surg 64: 1553-1561. 